\title{
Institutional Practice Guidelines on Management of Pelvic Fracture-Related Hemodynamic Instability: Do They Make a Difference?
}

\author{
Zsolt Balogh, MD, Erica Caldwell, RN, Martin Heetveld, MD, Scott D'Amours, MD, Glen Schlaphoff, MBBCh, \\ Ian Harris, MBBS, and Michael Sugrue, MD
}

Background: The management of patients with hemodynamic instability related to pelvic fracture is a major challenge, with high morbidity and mortality. Evidencebased institutional practice guidelines (PG) were developed as a strategy to optimize the care of these patients. The aims of this study were to evaluate the adherence to the new PG and compare the outcomes before and after their implementation.

Methods: Major blunt trauma patients (Injury Severity Score [ISS] > 15) with hemodynamic instability (initial base deficit $>6 \mathrm{mEq} / \mathrm{L}$ or received $>6$ units of packed red blood cells [PRBCs] during the first 12 hours) related to pelvic fracture were investigated. Patients presenting with ongoing bleeding from other regions or with severe head injury (Glasgow Coma Scale score $<9)$ were excluded. The pre-PG group $(n=17)$ were patients managed during the 18 months ending on December 31, 2001. The post-PG group $(n=$
14) consisted of patients managed during the subsequent 18 months. Demographics, ISS, shock severity, resuscitation, and outcome data were prospectively collected. The adherence to the key steps of PG was evaluated retrospectively in the pre-PG and prospectively in the post-PG group, including abdominal clearance (AC) with diagnostic peritoneal aspiration/lavage or ultrasound ( $<15$ minutes), noninvasive pelvic binding (PB) (<15 minutes), pelvic angiography (PA) (<90 minutes after admission), and minimally invasive orthopedic fixation (MIOF) ( $<24$ hours). Data are presented as mean \pm SEM or percentages.

Results: The pre-PG and post-PG groups were similar regarding age $(40 \pm 4$ years vs. $42 \pm 6$ years), gender (both $71 \%$ male), ISS ( $39 \pm 3$ vs. $37 \pm 4$ ), admission base deficit ( $9 \pm 1$ vs. $10 \pm 1)$ admission systolic blood pressure (116 \pm 7 vs. $112 \pm$ $6 \mathrm{~mm} \mathrm{Hg})$, Glasgow Coma Scale score (12 \pm 1 vs. $12 \pm 1$ ), and PRBC transfusion in the first 12 hours $(9 \pm 2 \mathrm{U}$ vs. $9 \pm 2 \mathrm{U})$. The adherence to the guidelines in the post-PG period was as follows: $\mathrm{AC}, \mathbf{1 0 0 \%}$; PB, $86 \%\left(p<0.05\right.$ based on $t$ test or $\chi^{2}$ test); PA, $93 \%(p<0.05$ based on $t$ test or $\chi^{2}$ test); and MIOF, $86 \%$. In the pre-PG period, adherence to the guidelines was as follows: AC, $65 \%$; PB, $0 \%$; PA, 30\%; and MIOF $52 \%$. In the post-PG period, the 24-hour PRBC transfusion decreased from $16 \pm 2 \mathrm{U}$ to $11 \pm 1 \mathrm{U}$ and the mortality decreased from $35 \%$ to $7 \%(p<$ 0.05 based on $t$ test or $\chi^{2}$ test for both).

Conclusion: The adherence to the PG as a reflection of optimal management was significantly improved. PG focusing particular on timely hemorrhage control reduced the 24-hour transfusion requirements and the mortality rate in the post-PG group.

Key Words: Pelvic fracture, Practice guidelines, Pelvic hemorrhage, Pelvic trauma.

J Trauma. 2005;58:778-782.
$\mathbf{T}$ he management of patients with hemodynamic instability related to pelvic fracture is a multidisciplinary challenge, with mortality ranging between $10 \%$ and $42 \%{ }^{1-5}$ The high mortality is related to uncontrolled pelvic bleeding in more than $40 \%$ of the cases. ${ }^{6-8}$ Our recent review of the Liverpool Hospital's trauma registry revealed $37 \%$ mortality in this high-risk group. ${ }^{9}$ Evidence-based institutional practice guidelines (PG) were developed at our institution as a strategy to optimize the care of hemodynamically unstable patients with pelvic fractures to significantly improve the management of these patients. ${ }^{10,11}$ The aims of this study were to

Submitted for publication July 27, 2004.

Accepted for publication January 13, 2005

Copyright () 2005 by Lippincott Williams \& Wilkins, Inc.

From the Departments of Trauma (Z.B., E.C., M.H., S.D., M.S.), Orthopaedics (E.H.), and Interventional Radiology (G.S.), Liverpool Health Service, Sydney, New South Wales, Australia.

Address for reprints: Michael Sugrue, MD, Department of Trauma, Locked Bag 7103, Liverpool BC, NSW 1871, Australia; email: michael.sugrue@swsahs.nsw.gov.au.

DOI: $10.1097 / 01 . T A .0000158251 .40760 . B 2$ evaluate adherence to the new PG and compare the outcomes before and after their implementation.

\section{PATIENTS AND METHODS}

Trauma patients with pelvic fracture and hemodynamic instability managed in the Liverpool Hospital, which is the university-affiliated major trauma center of South Western Sydney with annual trauma admissions of more 2,200 and serving a population of roughly 1 million. During the 36month period ending in July 2003, there were 6,882 trauma admissions and 354 pelvic fracture patients admitted to Liverpool Hospital. Major blunt trauma patients with an Injury Severity Score (ISS) $>15$ were included in the study. Other inclusion criteria were the presence of hemorrhagic shock defined by the initial base deficit higher than $6 \mathrm{mEq} / \mathrm{L}$ and more than $6 \mathrm{U}$ of packed red blood cells (PRBCs) transfused during the first 12 hours. Major head injury patients (defined by a Glasgow Coma Scale score $<9$ ) and patients with uncontrolled extrapelvic bleeding were excluded. We evaluated the adherence to the newly implemented PG and com- 
Table 1 Demographics, Injury, and Shock Severity of the Pelvic Trauma Patients before and after Implementation of the Practice Guidelines

\begin{tabular}{|c|c|c|c|c|c|c|c|}
\hline Group & Age (yr) & Male (\%) & ISS & $\mathrm{BD}(\mathrm{mEq} / \mathrm{L})$ & $\mathrm{SBP}(\mathrm{mm} \mathrm{Hg})$ & GCS Score & PRBCs (U/12 h) \\
\hline Pre-PG & $40 \pm 4$ & 71 & $39 \pm 3$ & $9 \pm 1$ & $116 \pm 7$ & $12 \pm 1$ & $9 \pm 2$ \\
\hline Post-PG & $42 \pm 6$ & 71 & $37 \pm 4$ & $10 \pm 1$ & $112 \pm 6$ & $12 \pm 1$ & $9 \pm 2$ \\
\hline
\end{tabular}

Pre-PG, before implementation of the practice guidelines; Post-PG, after implementation of the practice guidelines; ISS, Injury Severity Score; BD, base deficit; SBP, systolic blood pressure; GCS, Glasgow Coma Scale; PRBCs, packed red blood cells; U/12 h, the number of transfused units during the first $12 \mathrm{~h}$.

pared the outcomes of patients between the two 18-month periods before and after January 2002.

From January 2002, evidence-based institutional PG were implemented to provide optimal care of this high-risk group. The PG were developed by a multidisciplinary group- under the supervision of the Injury Advisory Committee of the South Western Sydney Area Health Servicethat included trauma surgeons, an orthopedic trauma surgeon, an interventional radiologist, an emergency physician, an intensive care specialist, prehospital care providers, and nurse representatives of the listed specialties. The guidelines were widely disseminated to regional experts for peer review. From the prehospital notification through the technique of noninvasive pelvic binding, pelvic angioembolization, and minimally invasive orthopedic fixation (MIOF), all steps of the care of this group became standardized based on the available evidence in the literature.

This study focuses on the patients whose hemodynamic instability was related to pelvic bleeding only. According to the PG, the most important interventions with set timeframes were the following for this group: abdominal clearance (AC) with diagnostic peritoneal aspiration or focused abdominal sonography for trauma within 15 minutes; noninvasive pelvic binding $(\mathrm{PB})$ with a bed sheet or a proprietary device within 15 minutes; pelvic angiography (PA) within 90 minutes; and MIOF (external fixator, C-clamp, or percutaneous screw fixation, depending on the fracture pattern) within 24 hours if the patient's general condition allowed.

Information regarding patient demographics, injury severity, shock severity, and outcomes were prospectively collected in the trauma registry. The adherence to the key interventions of the guidelines (AC, PB, PA, and MIOF) was evaluated retrospectively during the pre-PG period and prospectively during the post-PG period.

Data are presented as mean \pm SEM in the text and tables. Data were analyzed using $\chi^{2}$ and $t$ tests. The $\chi^{2}$ test was used to compare ISS of the pre-PG and post-PG cohorts, a nonparametric scale assessment; $t$ tests (unpaired, two-tailed) were used to compare parametric quantities (e.g., base deficit measurements, PRBC volumes, and stay times). A difference between the pre-PG and post-PG cohorts with $p<0.05$ was considered significant.

\section{RESULTS}

During the 36-month study period, 31 patients met the inclusion criteria, of whom 17 were resuscitated during the pre-PG period (6 with anteroposterior compression, 2 with lateral compression, 7 with vertical shear, and 2 with a combined mechanism) and 14 during the post-PG period ( 3 with anteroposterior compression, 2 with lateral compression, 8 with vertical shear, and 1 with a combined mechanism). Demographics, injury, and shock severity data are summarized in Table 1. Pre- and Post-PG cohorts were not different regarding age, gender, injury mechanism, ISS, or severity of shock. Both groups required massive resuscitation in response to their initial shock. The adherence to the guidelines improved in the post-PG group (Table 2), the time of AC was optimized (100\%), the PB $<15$ minutes and PA $<90$ minutes significantly improved. Sixteen of the 17 (93\%) post-PG period patients had PA within 90 minutes. There was a trend toward a higher percentage of patients receiving MIOF $<24$ hours in the post-PG group and, whereas in the pre-PG group, MIOF meant exclusively external fixation (in all nine cases) regardless of the injury pattern. During the post-PG period, external fixation alone was used only in anteroposterior compression type fractures ("open book"). In cases where vertical instability was present with disruption of the posterior elements of the pelvic ring, C-clamp (two cases) or percutaneous iliosacral screw insertion (seven cases) was preferred as early intervention. Pre- and post-PG patients had similar intensive care unit length of stay and multiple organ failure (MOF) (defined by the Denver MOF score) incidence

\section{Table 2 Adherence to the Practice Guidelines and Outcome of the Pelvic Fracture Patients}

\begin{tabular}{ccccccccc}
\hline Group & AC (\%) & PB (\%) & PA (\%) & MIOF (\%) & PRBC U/24 h & ICU LOS (days) & MOF (\%) & Mortality (\%) \\
\hline Pre-PG & 65 & 0 & 30 & 52 & $16 \pm 2$ & $6 \pm 2$ & 18 & 35 \\
Post-PG & 100 & $86^{*}$ & $93^{*}$ & 86 & $11 \pm 1^{\star}$ & $8 \pm 1$ & 14 & $7^{\star}$ \\
\hline
\end{tabular}

${ }^{*} p<0.05$ based on $t$ test or $\chi^{2}$ test.

Pre-PG, before implementation of the practice guidelines; Post-PG, after implementation of the practice guidelines; $A C$, abdominal clearance; PB, pelvic binding; PA, pelvic angiography; MIOF, minimally invasive orthopedic fixation; PRBCs, packed red blood cells; U/24 h, the number of transfused units during the first $24 \mathrm{~h}$; ICU LOS, intensive care unit length of stay. 
but with similar shock and injury severity, and the pre-PG patients required transfusion of more PRBCs during the first 24 hours and experienced a significantly higher mortality rate (Table 2). Six patients (35\%) died in the pre-PG group (four exsanguinated and two died as a result of MOF); the only death $(7 \%)$ in the post-PG period was caused by MOF.

\section{DISCUSSION}

Timely identification and control of pelvic hemorrhage is pivotal to decrease pelvic fracture-related mortality. ${ }^{12-16}$ The possible sources of pelvic blood loss are venous, arterial, and cancellous bone. ${ }^{17}$ Apart from the pelvic-related sources, abdominal bleeding is present in $35 \%$ of the major pelvic fractures. ${ }^{18}$ The Liverpool Hospital's pelvic PG was developed to provide evidence-based decision support to optimize outcome of hemodynamically unstable pelvic fracture patients. ${ }^{9-11}$ The optimal care-adherence to the PG-was achievable according to our results, and the outcomes improved after implementation of the PG.

Abdominal clearance is an early crucial point in the management of this group, given the high chance of pelvic and/or abdominal bleeding. Either focused abdominal sonography for trauma or diagnostic peritoneal aspiration should be performed within 15 minutes after admission, and the results of these tests are essential in triaging unstable patients between the operating room (laparotomy) or the angiography suite (pelvic embolization). ${ }^{19-23}$ During the pre-PG period, only two thirds of the patients had initial abdominal diagnostics within 15 minutes, but in the post-PG period, this reached $100 \%$, although the increase is not significant. The abdominal clearance was performed in all post-PG patients, which means that in this part of the management, complete adherence was achieved.

After identifying the pelvis as the source of hemorrhage (by physical examination, pelvic radiograph, and exclusion of the other regions), all possible pelvic sources of bleeding should be addressed. The role of pelvic binding in the reduction of the pelvic volume and providing temporary relative stability and consequent decrease in venous bleeding has not yet been proven. However, noninvasive pelvic binding with a readily available bed sheet or with a proprietary device is a safe and potentially beneficial step that can be performed very early without specialist involvement. ${ }^{24}$ During our pre-PG period, PB was not used in the management of unstable pelvic fracture patients. This newly implemented intervention was performed within 15 minutes in $86 \%$ of the patients during the post-PG period. In two cases, the PB was not used because the fracture pattern (lateral compression injury) did not suggest any additional benefit from the closure of the pelvic ring. The impact of PB on the outcome of pelvic bleeding needs to be clarified in the future.

No randomized trials are available to compare PA with other possible methods of hemorrhage control such as skeletal stabilization and/or pelvic retroperitoneal packing. However, on the basis of clinical series and expert opinion, PA is considered by most authors to be the optimal method for controlling arterial hemorrhage. ${ }^{25-28}$ The success of PA and embolization is determined by the availability of a skilled interventional radiologist and timely identification of patients who have significant pelvic arterial bleeders controllable with embolization. Contrast blush on computed tomographic (CT) scanning is a very specific sign of arterial bleeding, ${ }^{29,30}$ but even with the newer generation of CT scanners, transfer of hemodynamically unstable patients to the CT scanner, where hemorrhage control cannot be performed, is still questionable. Fracture pattern is reported to have good sensitivity but poor specificity for predicting the presence of significant arterial bleeders. ${ }^{31-33}$ The presence of hemorrhagic shock without nonpelvic bleeding sources, transient responsiveness, and nonresponsiveness to the initial resuscitation with persistent acidosis requiring massive resuscitation are the most useful predictors of potentially therapeutic angioembolization. ${ }^{30}$ Following this rationale, hemodynamically unstable patients with pelvic fracture and without other obvious sources of bleeding all should undergo PA within 90 minutes, according to the PG. With the implementation of the PG during the post-PG period, PA was performed within 90 minutes in all but one (93\%) study patient; in the pre-PG period, the rate was $30 \%$. This statistically significant improvement was the most difficult to achieve. In our institution, it was accomplished by widespread dissemination of the PG among the involved subspecialties, involvement of dedicated trauma surgeons to the resuscitations, regular case discussions on the weekly trauma audits, and positive feedback given to all trauma caregivers. In addition, the contribution of the interventional radiologist with special interest in trauma was essential to facilitate the well-timed interventions.

Bleeding from cancellous bony surfaces occurs in all pelvic fractures to some extent, depending on the displacement and the size of the fractured surfaces. In isolated lowenergy fractures, this bleeding has a self-limiting nature, but in high-energy cases where severe shock is present and by the arterial hemorrhage control acidosis, hypothermia, and coagulopathy exist, bleeding from cancellous bony surfaces became more significant. Closed pelvic reduction is easier in the acute setting, and minimally invasive pelvic fixation (with external fixator, C-clamp, or percutaneous screws, depending on the fracture pattern) could minimize bone-associated bleeding. ${ }^{34-37}$ Furthermore, early fixation helps positioning of the patients for ventilation, decreases pain and discomfort, and facilitates mobilization. Early definitive pelvic fixation (within 24 hours, according to our PG) should be used very judiciously, depending on the patient's general condition and ideally performed during other team's work in the operating room. With the active involvement of our orthopedic trauma team, we managed to increase the 24-hour MIOF rate in the post-PG period and adjust fixation methods to the injury pattern. ${ }^{38-40}$ Given the fact that most but not all pelvic fractures with hemodynamic instability require operative fixation, the $86 \%$ MIOF rate achieved in the post-PG period is unlikely to need further improvement. The potential benefits 
of earlier pelvic fixation and their relation to the outcome needs to be clarified and supported with further clinical research.

Numerous clinical pathways were developed and published during the last 20 years for management of hemodynamically unstable patients. Most of them consist of abdominal diagnostics, pelvic binding and/or external fixation, pelvic retroperitoneal packing, angioembolization, and orthopedic fixation of the pelvis. None of the listed interventions' efficacy in reducing mortality is proven by level I investigations, but all of the studies concluded that the prospective multidisciplinary awareness improves the outcome of these high-risk patients on the single-institutional level. ${ }^{21}$ Most of the protocols are institution specific, applied to the local environment of availability and responsiveness of different specialists such as general surgery, orthopedic surgery, interventional radiology, emergency medicine, and intensive care. Trauma care providers in historically different trauma care systems (i.e., German vs. Anglo-American systems) have varied levels of interest and expertise in the management of the injured pelvis. In Germany and in countries following the German system where comprehensive trauma surgeons (traumatologists) are responsible for orthopedic trauma, pelvic fractures tend to be fixed earlier and large expanding retroperitoneal hematomas are managed with packing against the restored pelvic ring. ${ }^{41}$ In the United States, where general surgical trauma surgeons are responsible for the resuscitation, life-saving surgical interventions, and critical care of severely injured patients, orthopedic surgeons are consulting specialists that deal only with the musculoskeletal injuries. In this system, arterial bleeding in hemodynamically unstable patients is embolized or excluded in the angiography suite before definitive pelvic fixation is performed by the consulting orthopedic trauma specialists. ${ }^{21,25,26}$ In both systems, emergency pelvic stabilization is performed with various methods and locations and with different timeframes. Both approaches could work highly efficiently in its setting but has never been compared in a multi-institutional randomized fashion.

The Liverpool Hospital pelvic trauma PG focusing on localization and control of pelvic bleeding significantly decreased the first-24-hour transfusion rate, whereas the amount of PRBCs transfused during the first 12 hours was the same in the pre-PG and post-PG groups. The amount of blood transfusion is an independent predictor of postinjury multiple organ failure (MOF), which is the major contributor of postinjury death among patients surviving the initial insult. ${ }^{42}$ In our relatively small series, the decreased amount of PRBCs transfused in the first 24 hours did not result in a decreased incidence of MOF. The adherence to the PG was reflected also in improved mortality in the post-PG group, which is comparable to the most favorable outcomes published in the literature among hemodynamically unstable pelvic fracture patients. In addition to the decreased mortality rate, no patient exsanguinated in the post-PG period.
The Liverpool pelvic trauma PG is a simple and effective time goal-oriented protocol that could be performed at any trauma center where interventional radiology is available. Our study proved that adherence to the guidelines can be achieved and the mortality rate can be decreased from $35 \%$ to $7 \%$. The effectiveness of our guidelines needs to be evaluated in other centers. In the future, apart from evaluating different protocols in multicenter studies, it is essential to determine the impact of individual interventions used during the management of severe pelvic fracture patients on effective hemorrhage control and outcome.

\section{REFERENCES}

1. Dalal SA, Burgess AR, Siegel JH, et al. Pelvic fracture in multiple trauma: classification by mechanism is key to pattern of organ injury, resuscitative requirements and outcome. J Trauma. 1989; 29:981-1002.

2. McMurtry R, Walton D, Dickenson D, Kellam J, Tile M. Pelvic disruption in the polytraumatized patient. Clin Orthop. 1980;151:2230 .

3. Moreno C, Moore EE, Rosenberger A, Cleveland HC. Haemorrhage associated with major pelvic fracture. J Trauma. 1986;26:987-989.

4. Rothenberger DA, Fischer RP, Strate RG, Velasco R, Perry JF Jr. The mortality associated with pelvic fractures. Surgery. 1978; 84:356-361.

5. Naam NH, Brown WH, Hurd R, Burdge RE, Kaminski DL. Major pelvic fractures. Arch Surg. 1983;118:610-616.

6. Evers BM, Cryer HM, Miller FB. Pelvic fracture haemorrhage. Arch Surg. 1989;124:422-424.

7. Poole GV, Ward EF. Causes of mortality in patients with pelvic fractures. Orthopedics. 1994;17:691-696.

8. Gilliland MD, Ward RE, Barton RM, Miller PW, Duke JH. Factors affecting mortality in pelvic fractures. J Trauma. 1982;22:691-693.

9. Heetveld MJ, Harris I, Balogh Z, Schlaphoff G, D’Amours SK, Sugrue M. Haemodynamically unstable pelvic fractures: recent care and new guidelines. World J Surg 2004;28:904-909.

10. Heetveld MJ, Harris I, Schlaphoff G, Sugrue M. Guidelines for the management of hemodynamically unstable pelvic fracture patients. Aust N Z J Surg. 2004;74:520-529.

11. Heetveld MJ, Harris I, Schlaphoff G. Pelvic fracture algorithm. In: D’Amours SK, Sugrue M, Russell R, Nocera N, eds. Handbook of Trauma Care. Liverpool Hospital Trauma Manual. 6th ed. Sydney: Graphitype; 2002.

12. Mucha P, Farnell MB. Analysis of pelvic fracture management. J Trauma. 1984;24:379-386.

13. Panetta T, Sclafani SGA, Goldstein AS, Phillips TF, Shaftan GW. Percutaneous transcatheter embolisation for massive bleeding from pelvic fractures. J Trauma. 1985;25:1021-1029.

14. Flint LM, Babikian G, Anders M, Rodriquez J, Steinberg S. Definitive control of mortality from severe pelvic fracture. Ann Surg. 1990;211:703-707.

15. Gruen GS, Leit ME, Gruen RJ, Peitzman AB. The acute management of hemodynamically unstable multiple trauma patients with pelvic ring fractures. J Trauma. 1994;36:706-713.

16. The Eastern Association for the Surgery of Trauma website. Available at: http://www.east.org/tpg/pelvis.pdf. Accessed September $16,2003$.

17. Huittinen VM, Slätis P. Post mortem angiography and dissection of the hypogastric artery in pelvic fractures. Surgery. 1973;73:454-462.

18. Hawkins L, Pomerantz M, Eisman B. Laparotomy at the time of pelvic fracture. J Trauma. 1970;10:619-623.

19. Sugrue M. Diagnostic peritoneal aspiration (DPA) and diagnostic peritoneal lavage (DPL). In: D’Amours SK, Sugrue M, Russell R, 
Nocera N, eds. Handbook of Trauma Care. Liverpool Hospital Trauma Manual. 6th ed. Sydney: Graphitype; 2002;161-165.

20. Sugrue M, Seger M, Gunning K, Sloane D, Deane S. A modified combination technique for performing diagnostic peritoneal lavage. Aust N Z J Surg. 1995;65:604-606.

21. Biffl WA, Smith WR, Moore EE, et al. Evolution of a multidisciplinary clinical pathway for the management of haemodynamically unstable patients with a pelvic fracture. Ann Surg. 2001;233:843-850.

22. Nunes LW, Simmons S, Hallowell MJ, Kinback R, Trooskin S, Kozar R. Diagnostic performance of trauma US in identifying abdominal or pelvic free fluid and serious abdominal or pelvic injury. Acad Radiol. 2001;8:128-136.

23. Rozycki GS, Ballard R, Feliciano DV, Schmidt JA, Pennington SD. Surgeon performed ultrasound for the assessment of truncal injuries. Ann Surg. 1998;228:557-567.

24. Simpson T, Krieg JC, Heuer F, Bottlang M. Stabilization of pelvic ring disruptions with a circumferential sheet. J Trauma. 2002; 52:158-161.

25. Agolini SF, Shah K, Jaffe J, Newcomb J, Rhodes M, Reed JF. Arterial embolisation is a rapid and effective technique for controlling pelvic fracture haemorrhage. J Trauma. 1997;43:395399.

26. Velmahos GC, Toutouzas K, Vassiliu P, et al. A prospective study on the safety and efficacy of angiographic embolization for pelvic and visceral injuries. J Trauma. 2002;53:303-308.

27. Kaufman JA, Waltman AC. Angiographic management of haemorrhage in pelvic fractures. In: Baum M, Pentecost E, eds. Abrams' Angiography : Interventional Radiology. Philadelphia: Lippincott-Raven; 1996:869-883.

28. Perez JV, Hughes PMD, Bowers SK. Angiographic embolisation in pelvic fracture. Injury. 1998;29:187-191.

29. Shanmuganathan K, Mirvis SE, Sover ER. Value of contrast enhanced CT in detecting active haemorrhage in patients with blunt abdominal or pelvic trauma. AJR Am J Roentgenol. 1993;161:65-69.
30. Miller PR, Moore PS, Mansell E, et al. External fixation or arteriogram in bleeding pelvic fracture: initial therapy guided by markers of arterial hemorrhage. J Trauma. 2003;54:437-443.

31. Cryer HM, Miller FB, Evers EM, Rouben LR, Seligson DL. Pelvic fracture classification: correlation with haemorrhage. J Trauma. 1988;28:973-980.

32. Eastridge BJ, Starr A, Minei JP, O'Keefe GE. The importance of fracture pattern in guiding therapeutic decision-making in patients with hemorrhagic shock and pelvic ring disruptions. J. Trauma. 2002;53:446-451

33. Hamill J, Holden A, Paice R, Civil I. Pelvic fracture pattern predicts arterial hemorrhage. Aust N Z J Surg. 2000;70:338-343.

34. Riemer BL, Butterfield SL, Diamond DL, et al. Acute mortality associated with injuries to the pelvic ring: the role of early patient mobilization and external fixation. J Trauma. 1993;35:671-677.

35. Tile M. Pelvic ring fractures: should they be fixed? J Bone Joint Surg Br. 1988;70:1-12.

36. Kellam JF. The role of external fixation in pelvic disruptions. Clin Orthop. 1989;241:66-82.

37. Ganz R. The antishock pelvic clamp. Clin Orthop. 1991;267:71-78.

38. Routt MLC, Simonian PT, Ballmer F. A rational approach to pelvic trauma. Clin Orthop. 1995;318:61-74.

39. Latenser BA, Gentillello LM, Tarver AA, Thalgott JS, Batdorf JW. Improved outcome with early fixation of skeletally unstable pelvic fractures. J Trauma. 1991;31:28-31.

40. Waikakul S, Harnroonroj T, Vanadurongwan V. Immediate stabilization of unstable pelvic fractures vs delayed stabilization. J Med Assoc Thai. 1999;82:637-642.

41. Ertel W, Keel M, Eid K, Platz A, Trentz O. Control of severe hemorrhage using C-clamp and pelvic packing in multiply injured patients with pelvic ring disruption. J Orthop Trauma. 2001;15:468474.

42. Moore FA, Moore EE, Sauaia A. Blood transfusion: an independent risk factor for postinjury multiple organ failure. Arch Surg. 1997; 132:620-625. 